\title{
NATIONAL INSURANCE ACT.
}

Report By Mir. G. F. Hardy, F.I.A., and Mr. F. B. WyatT, F.I.A., on the Revision or Extension of the Original Estimates, caused by Changes Effected in Committee in Part I of THE BILL.

TO THE LORDS COMMISSIONERS OF HIS MAJESTY'S TREASURY.

May it Please Your Lordships,

28 November 1911.

1. We have at your Lordships' request revised certain tables and estimates contained in our Report of the 20 May last upon the case submitted to us in connection with the National Insurance Bill as introduced, and have made such corrections therein as are rendered necessary by the changes embodied in the Bill as it left the Committee on the 21 st instant. The changes effected in the Bill, which render necessary a revision or extension of our original estimates, are :

(1) Those relating to the population coming within the scope of the scheme.

(2) Those involving separate estimates for England, Wales, Scotland and Ireland in lieu of the original estimates for the United Kingdom as a whole.

(3) The alterations that have been made in the benefits payable to insured persons.

\section{Population coming under the Scheme.}

2. As regards the population included in the Bill the principal change is the omission of young persons under 16 and the inclusion as voluntary insured persons, upon a reduced scale of contributions and benefits, of married women, not being "employed persons", 
who have been contributors prior to marriage. As regards young persons under 16 these were separately dealt with in our Report of the 20 May and their exclusion will not affect the tables in that report showing the estimated number of compulsory and voluntary contributors as at 1 May 1912. It is now proposed that the Act should come into operation at a later date, and we have, for the purpose of estimating the income and expenditure under the Bill, assumed, in accordance with instructions, that this date will be the 1 July 1912. We have not, however, considered it necessary to re-estimate the number of contributors at the outset, as a postponement for a period of two months will not materially affect these. The estimated numbers in the case of England and Wales might be increased by about 2 per 1,000 , in the case of Scotland by about 1 per 1,000 , while in the case of Ireland, as we have assumed the population to be stationary, there would be no change.

3. Tables $I$ to $V$ appended give separately for England, Wales, Scotland, and Ireland, the estimated number of persons becoming members of approved societies at the commencement of the scheme, distinguishing between compulsory and voluntary contributors, and in the case of women between spinsters, married, and widows. The estimated number of members of approved societies (compulsory and voluntary) in future years are shown in Table VI. The figures in Tables I to VI do not include any estimate in respect of married women who may become voluntary contributors under clause 41 , as there is no data by which the probable number of these can be calculated. The figures are also exclusive of the Army and Navy, as there are no means of estimating how many of those in the Services will become members of approved societies. The estimated number of contributors in the Services in various groups is stated in our Report of 26 July last (Table D.).

4. As regards the deposit contributors, we estimate the numbers at the commencement of the scheme, as follows:

\begin{tabular}{|c|c|c|c|c|c|}
\hline & & & Men & Women & Both Sexes \\
\hline $\begin{array}{l}\text { England } \\
\text { Wales } \\
\text { Seotland } \\
\text { Ireland. }\end{array}$ & $\dot{\cdot}$ & . $\quad$. & $\begin{array}{r}499,000 \\
32,000 \\
72,000 \\
35,000\end{array}$ & $\begin{array}{r}186,000 \\
11,000 \\
26,000 \\
21,000\end{array}$ & $\begin{array}{r}685,000 \\
43,000 \\
98,000 \\
56,000\end{array}$ \\
\hline \multicolumn{2}{|c|}{ United Kingdom } & . & 638,000 & 244,000 & 882,000 \\
\hline
\end{tabular}

As pointed out in paragraph 66 of our Report of 20 May last, it is impossible to make an estimate of any value as to the probable number of deposit contributors in future years.

Benefits.

5. The " minimum benefits" provided by the Bill, in the case of fully insured persons, are set out in Schedule 4 of the Bill. They 
differ from the benefits provided by the Bill in its original form in the following particulars.

Sickness Benefit.-The allowance of $10 \mathrm{~s}$. a week in the case of men and $7 s .6 d$. a week in the case of women during the first 13 weeks of sickness, reckoning from the fourth day, is now extended to the first 26 weeks of sickness, reckoning from the fourth day. We have not, for the purpose of these estimates, taken account of the options specified in the Schedule, as the value of the alternative benefits is, on the average, nearly the same as that of the benefits above mentioned.

This allowance is reduced in the case of unmarried persons under 21 , in the case of men, to $6 s$. a week during the first 13 weeks and $5 s$. a week during the second 13 weeks, and, in the case of women, to $5 s$. a week and $4 s$. a week respectively for these periods.

It is also reduced in the case of persons who are between the ages of 50 and 60 at the time of becoming employed contributors, in the case of men to $7 s$. a week for the whole period of 26 weeks, and in the case of women to $6 s$. a week for the whole period, and for persons over 60 at the time of becoming employed contributors, in the case of both men and women to $6 s$. a week for the first 13 weeks and $5 s$. a week during the second 13 weeks.

Maternity Benefit.-This benefit is now extended to the wife of an insured person notwithstanding that she is herself an insured person.

The medical and sanatorium benefits remain unchanged, and according to our original instructions have been taken as equivalent to $6 s$. per head per annum, and $1 s .3 d$. per head per annum respectively throughout life.

\section{Contributions.}

6. The rates of contribution necessary to provide the benefits for persons entering insurance at age 16, we now estimate as follows :

TABLE A.

Contributions at Age 16 for Minimum Benefit.

\begin{tabular}{|c|c|c|c|c|}
\hline \multirow{2}{*}{ Benefit } & \multicolumn{2}{|c|}{ Pounds per ANNTM } & \multicolumn{2}{|c|}{$\begin{array}{c}\text { PENCE PER WHEK } \\
\text { CORRESPONDING THERETO }\end{array}$} \\
\hline & Men & Women & Men & Women \\
\hline $\begin{array}{l}\text { (a) Medical : } \\
\text { (b) Sanatorium : } \\
\text { (c) Sickness : } \\
\text { (d) Disablement : } \\
\text { (e) Maternity. }\end{array}$ & $\begin{array}{l}\stackrel{E}{*} \\
.328 \\
-070 \\
.519 \\
.170 \\
.143\end{array}$ & $\begin{array}{l}\qquad \\
.328 \\
.070 \\
.378 \\
.175 \\
.039\end{array}$ & $\begin{array}{r}d . \\
1 \cdot 51 \\
\cdot 32 \\
2 \cdot 39 \\
\cdot 78 \\
\cdot 66\end{array}$ & $\begin{aligned} d . \\
1 \cdot 51 \\
\cdot 32 \\
1 \cdot 74 \\
\cdot 81 \\
\cdot 17\end{aligned}$ \\
\hline $\begin{array}{l}\text { Total benefits } \\
\text { Cost of administration }\end{array}$ & $\begin{array}{r}1 \cdot 230 \\
\cdot 200\end{array}$ & $\begin{array}{l}.990 \\
.200\end{array}$ & $\begin{array}{r}5 \cdot 66 \\
.92\end{array}$ & $\begin{array}{r}4: 55 \\
\cdot 92\end{array}$ \\
\hline Total . & $1 \cdot 430$ & $1 \cdot 190$ & 6.58 & $5 \cdot 47$ \\
\hline
\end{tabular}


These contributions are to be payable up to age 70 , and are computed on the assumption that they will not be payable during the sickness or unemployment of the contributor.

7. In estimating these contributions the same assumptions have been made as to the rates of mortality and sickness as in our previous Report. As stated in paragraph 45 of that Report we consider that any saving that may be effected by the sickness benefit not commencing until the fourth day of sickness must be kept as a margin, and without this margin we do not consider that the rates of sickness employed in our calculations are applicable to the conditions of a national scheme as set out in the Bill. As regards the estimated cost of the medical and sanatorium benefits we are of opinion that no larger sum than that assumed, namely, $7 s .3 d$. per annum for these combined benefits, can safely be set aside out of the proposed contributions, and that the whole margin between the estimated contributions and those actually payable under the Bill should be regarded as available to meet the heavier rates of sickness and disablements which must be expected in a certain number of societies. Unless this margin is retained it is probable that a considerable number of societies will show deficiencies upon valuation, and this might endanger the success of the scheme.

\section{Initial Reserve Values.}

8. The aggregate initial reserves, computed in accordance with the principles explained in paragraphs 73 and 74 of our Report of 20 May last, we estimate as shown in the following Table B. These sums include the estimated capitalised liability in respect of women, married at the commencement of the scheme, who will eventually come into insurance during widowhood with title to full benefits.

TABLE B.

Estinated Aggregate Initial Reserve Values.

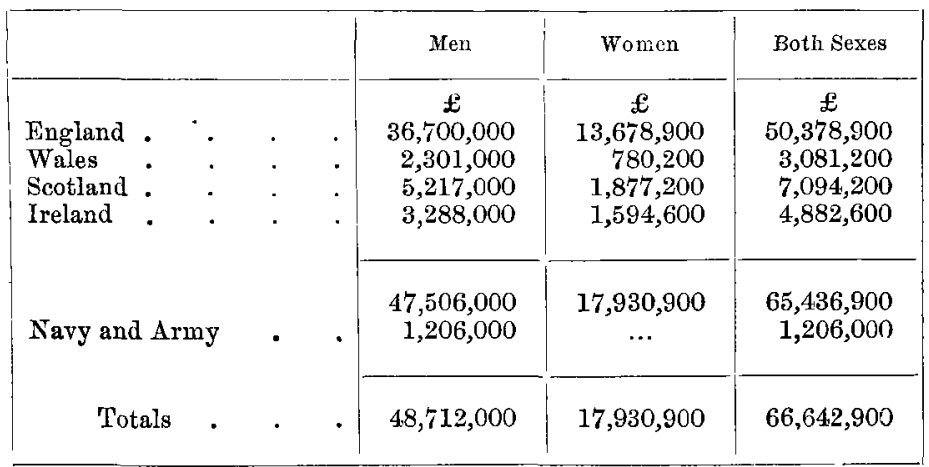


9. The estimated number of years required to liquidate the initial deficiencies represented by the above aggregate reserve values is as follows:

\begin{tabular}{|c|c|c|c|c|c|}
\hline \multicolumn{3}{|c|}{-} & Men & Women & Both Sexes \\
\hline $\begin{array}{l}\text { England } \\
\text { Wales } \\
\text { Scotland } \\
\text { Ireland }\end{array}$ & $\begin{array}{ll}\cdot & \cdot \\
\cdot & \cdot \\
\cdot & \cdot\end{array}$ & : & $\begin{array}{l}18 \frac{1}{2} \\
18 \frac{1}{18} \\
18 \frac{1}{4} \\
18 \frac{1}{2}\end{array}$ & $\begin{array}{l}17 \frac{1}{2} \\
17 \frac{1}{2} \\
16 \frac{1}{4} \\
19 \frac{3}{4}\end{array}$ & $\begin{array}{l}18 \frac{1}{4} \\
18 \frac{1}{2} \\
17 \frac{3}{4} \\
19\end{array}$ \\
\hline Uni & Kingdom & . & $18 \frac{1}{2}$ & $17 \frac{1}{2}$ & $18 \frac{1}{4}$ \\
\hline
\end{tabular}

In computing these terms of years the figures for the Navy and Army have been included in the figures for England.

\section{INCOME FROM CONTRIBUTIONS.}

10. The estimated aggregate contributions to be received from employers and insured persons who are members of approved societies in successive years is shown for each country in Table VII. The figures there given, however, include the special contributions of $1 d$. per week per head, to be paid by the State in the cases of employed persons whose wages are under $2 s$. a day, as provided in clause 6 of the Bill.

\section{Annual Cost of Benefits.}

11. Tables VIII to XI show the estimated annual cost in respect of the "minimum benefits" for England, Wales, Scotland, and Ireland. These figures do not include the sums that will be payable in respect of the maternity benefit in the case of the Navy and Army, and which may be taken approximately at $£ 28,000$ per annum.

The estimated annual cost of the "maximum benefits", including cost of administration, is shown in Table XII. The principle upon which these "maximum benefits" are determined is explained in paragraph 82 in our Report of 20 May last. As in the case of the "minimum" benefits they do not include the figures for the Navy and Army.

\section{Married Women Voluntary Insurers.}

12. Neither the statement of contributions given in Table VII, nor the statements of the cost of benefits in Tables VIII to XII take any account of those women contributors who, upon marriage, may elect to continue insurance under clause 41, upon the reduced scale of contributions and benefits provided for this class. There are, obviously, no means of estimating what proportion of women will so elect to continue insured, but the number may be considerable. These insurances will not affect the amount of the initial deficiencies represented by the aggregate reserve values in Table B, nor the terms required to liquidate these deficiencies. They will, however, 
affect the amount of the annual State subsidy, representing onefourth of the annual payments for "maximum" benefits.

On the basis of the rates of marriage of spinsters and widows respectively, given in our Report of 20 May last, the annual number of marriages among contributors to the scheme, where the married woman is not an "employed person", will be approximately 150,000, and if in one-half of these cases the contributor elects to continue insured under clause 41 there will, in the course of 10 years, be nearly 700,000 such insurances subsisting, representing an annual income from contributions of about $£ 450,000$, and an expenditure on account of benefits of somewhat less than this sum.

\section{Amount of State Subsidy.}

13. The estimated annual charge in respect of the State proportion of the " maximum " benefits and cost of administration in the four countries is shown in Table XIII. The figures there given are exclusive of those having reference to the Navy and Army and to the additional State contributions of $1 d$. a week in the case of employed persons whose wages are under $2 s$. a day. These sums are, however, given in Table XIV showing the annual amounts of the State subsidy for the United Kingdom. The figures for the additional contributions in respect of low-pald labour have been deduced from figures furnished to us by the Local Government Board, but we have no means of distributing them among the four countries.

14. The amount of the State subsidy, as shown in Table XIV, will also be increased by the State proportion of the benefits paid out to deposit contributors and of the benefits paid to married women voluntarlly insured. As regards the first of these items it is not practicable to determine what proportion of the contributions paid in to the Post Office by deposit contributors will be claimed in the form of benefits. In the early years of the scheme's operation the sums so paid out must necessarily be less, and may be considerably less, than the contributions pald in, but in later years the outgo may very well reach the level of the income, or possibly exceed it. As already stated, however, there are no means by which the number of deposit contributors in future years can be estrmated. On the basis of the estimated numbers at the commencement of the scheme, as given on page 1 of the Report, it would seem probable that the annual cost to the State in respect of deposit contributors will not exceed $£ 250,000$ per annum, and is not likely to exceed about half that sum in the first financial year.

15. The cost of the State proportion of benefits in the case of married women voluntary insurers has not been included in Table XIV owing to the impossibility of making any trustworthy estimate. In the nature of the case the cost must be insignificant at the outset, but, on the assumption that one-half of those who upon marriage are entitled to remain assured upon the reduced scale of benefits will so elect, the cost to the State 10 years from the commencement of the scheme may reach about $£ 150,000$ per annum, the exact sum depending not only upon the numbers exercising their option to remain insured but also upon their average age at marriage. 
16. If rough estimates of the cost made in the above manner of the State proportion of benefits in the case of deposit contributors and married women voluntary insurers may be taken as reasonable, the result will be to raise the estimated cost to the State, as shown in Table XIV, approximately to the following amounts:

\section{Estimated Total Cost of State Subsidy.}

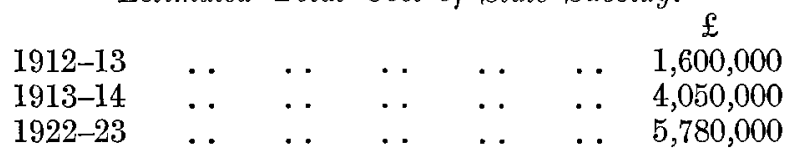

17. In submitting the above further estimates we wish to point out that they must be taken in connection with the various qualifications described in our Report of 20 May last.

We have the honour to be,

Your Lordships' obedient Servants,

GEORGE F. HARDY,
FRANK B. WYATT, 


\section{TABLE I.}

Estimated Number of Persons becoming Members of Approved Societies as at 1 May 1912.

ENGLAND.

Men.

\begin{tabular}{|c|c|c|c|}
\hline Ages & Compulsory & Voluntary & Both Classes \\
\hline $\begin{array}{l}16-20 \\
20-25 \\
25-30 \\
30-35 \\
35-40 \\
40-45 \\
45-50 \\
50-55 \\
55-60 \\
60-65\end{array}$ & $\begin{array}{r}917,000 \\
1,034,000 \\
944,000 \\
868,000 \\
769,000 \\
659,000 \\
545,000 \\
425,000 \\
310,000 \\
211,000\end{array}$ & $\begin{array}{l}11,300 \\
27,300 \\
44,200 \\
58,300 \\
69,600 \\
75,300 \\
35,800 \\
25,400 \\
16,900 \\
10,400\end{array}$ & $\begin{array}{r}928,300 \\
1,061,300 \\
988,200 \\
926,300 \\
838,600 \\
734,300 \\
580,800 \\
450,400 \\
326,900 \\
221,4,00\end{array}$ \\
\hline $60-65$ & $6,682,000$ & 374,500 & $7,056,500$ \\
\hline
\end{tabular}

Women.

\begin{tabular}{|c|c|c|c|c|c|c|c|c|}
\hline \multirow{2}{*}{ Ages } & \multicolumn{4}{|c|}{ COMPULSORY } & \multicolumn{3}{|c|}{ VoLUNTARY } & \multirow{2}{*}{$\begin{array}{c}\text { Both } \\
\text { Classes }\end{array}$} \\
\hline & Spinsters & Married & Widows & Total & Spinsters & Widows & Total & \\
\hline $16-20$ & 689,000 & 6,000 & & 695,000 & 18,900 & & 18,900 & 713,900 \\
\hline $20-25$ & 628,000 & 34,000 & 2,000 & 664,000 & 22,600 & 100 & 22,700 & 686,700 \\
\hline $25-30$ & 329,000 & 58,000 & 8,000 & 395,000 & 18,400 & 500 & 18,900 & 413,900 \\
\hline $30-35$ & 180,000 & 72,000 & 17,000 & 269,000 & 16,500 & 1,500 & 18,000 & 287,000 \\
\hline $35-40$ & 108,000 & 71,000 & 26,000 & 205,000 & 14,500 & 3,500 & 18,000 & 223,000 \\
\hline $40-45$ & 70,000 & 60,000 & 36,000 & 166,000 & 12,600 & 6,300 & 18,900 & 184,900 \\
\hline $45-50$ & 46,000 & 48,000 & 42,000 & 136,000 & 4,900 & 4,500 & 9,400 & 145,400 \\
\hline $50-55$ & 30,000 & 35,000 & 45,000 & 110,000 & 3,400 & 5,100 & 8,500 & 118,500 \\
\hline $55-60$ & 18,000 & 23,000 & 41,000 & 82,000 & 1,700 & 4,000 & 5,700 & 87,700 \\
\hline \multirow[t]{2}{*}{$60-65$} & 11,000 & 13,000 & 36,000 & 60,000 & 900 & 2,900 & 3,800 & 63,800 \\
\hline & $2,109,000$ & 420,000 & 253,000 & $2,782,000$ & 114,400 & 28,400 & 142,800 & $2,924,800$ \\
\hline
\end{tabular}


TABLE II.

Estimated Number of Persons becoming Members of Approved Societies as at 1 May 1912.

WALES.

Men.

\begin{tabular}{|c|c|c|c|}
\hline Ages & Compulsory & Foluntary & Both Classes \\
\hline \multirow[t]{2}{*}{$\begin{array}{l}16-20 \\
20-25 \\
25-30 \\
30-35 \\
35-40 \\
40-45 \\
45-50 \\
50-55 \\
55-60 \\
60-65\end{array}$} & $\begin{array}{l}57,000 \\
66,000 \\
59,000 \\
54,000 \\
48,000 \\
41,000 \\
34,000 \\
27,000 \\
20,000 \\
13,000\end{array}$ & $\begin{array}{r}700 \\
1,700 \\
2,800 \\
3,700 \\
4,400 \\
4,700 \\
2,200 \\
1,600 \\
1,100 \\
600\end{array}$ & $\begin{array}{l}57,700 \\
67,700 \\
61,800 \\
57,700 \\
52,400 \\
45,700 \\
36,200 \\
28,600 \\
21,100 \\
13,600\end{array}$ \\
\hline & 419,000 & 23,500 & 442,500 \\
\hline
\end{tabular}

Women.

\begin{tabular}{|c|c|c|c|c|c|c|c|c|}
\hline \multirow{2}{*}{ Ages } & \multicolumn{4}{|c|}{ CoMrolsory } & \multicolumn{3}{|c|}{ VOLUNTARY } & \multirow{2}{*}{$\begin{array}{c}\text { Both } \\
\text { Classes }\end{array}$} \\
\hline & Spinsters & Married & Widows & Total & Spinsters & Widows & Total & \\
\hline $16-20$ & 39,000 & & & 39,000 & 1,100 & $\cdots$ & 1,100 & 40,100 \\
\hline $20-25$ & 36,000 & 2,000 & ... & 38,000 & 1,300 & 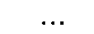 & 1,300 & 39,300 \\
\hline $25-30$ & 19,000 & 3,000 & & 22,000 & 1,100 & & 1,100 & 23,100 \\
\hline $30-35$ & 10,000 & 4,000 & 1,000 & 15,000 & 900 & 100 & 1,000 & 16,000 \\
\hline $35-40$ & 6,000 & 4,000 & 2,000 & 12,000 & 800 & 200 & 1,000 & 13,000 \\
\hline $40-45$ & 4,000 & 4,000 & 2,000 & 10,000 & 700 & 4.00 & 1,100 & 11,100 \\
\hline $45-50$ & 3,000 & 3,000 & 2,000 & 8,000 & 300 & 300 & 600 & 8,600 \\
\hline $50-55$ & 2,000 & 2,000 & 2,000 & 6,000 & 200 & 300 & 500 & 6,500 \\
\hline $55-60$ & 1,000 & 1,000 & 2,000 & 4,000 & 100 & 200 & 300 & 4,300 \\
\hline \multirow[t]{2}{*}{$60-65$} & 1,000 & 1,000 & 2,000 & 4,000 & $\ldots$ & 200 & 200 & 4,200 \\
\hline & 121,000 & 24,000 & 13,000 & 158,000 & 6,500 & 1,700 & 8,200 & 166,200 \\
\hline
\end{tabular}




\section{TABLE III.}

Estimated Number of Persons becoming Members of Approved Societies as at 1 May 1912.

Scotland.

Men.

\begin{tabular}{|c|c|c|c|}
\hline Ages & Compulsory & Voluntary & Both Classes \\
\hline $\begin{array}{l}16-20 \\
20-25 \\
25-30 \\
30-35 \\
35-40 \\
40-45 \\
45-50 \\
50-55 \\
55-60 \\
60-65\end{array}$ & $\begin{array}{r}148,000 \\
163,000 \\
143,000 \\
125,000 \\
110,000 \\
93,000 \\
75,000 \\
59,000 \\
44,000 \\
29,000\end{array}$ & $\begin{array}{l}2,000 \\
4,000 \\
5,000 \\
6,000 \\
7,000 \\
8,000 \\
4,000 \\
3,000 \\
2,000 \\
1,000\end{array}$ & $\begin{array}{r}150,000 \\
167,000 \\
148,000 \\
131,000 \\
117,000 \\
101,000 \\
79,000 \\
62,000 \\
46,000 \\
30,000\end{array}$ \\
\hline & 989,000 & 42,000 & $1,031,000$ \\
\hline
\end{tabular}

Women.

\begin{tabular}{|c|c|c|c|c|c|c|c|c|}
\hline \multirow{2}{*}{ Ages } & \multicolumn{4}{|c|}{ Compulsory } & \multicolumn{3}{|c|}{ Voluntary } & \multirow{2}{*}{$\begin{array}{c}\text { Both } \\
\text { Classes }\end{array}$} \\
\hline & Spinsters & Married & Widows & Total & Spinsters & Widows & Total & \\
\hline $16-20$ & 109,000 & 1,000 & $\ldots$ & 110,000 & 4,000 & $\ldots$ & 4,000 & 114,000 \\
\hline $20-25$ & 95,000 & 5,000 & & 100,000 & 5,000 & $\ldots$ & 5,000 & 105,000 \\
\hline 25-30 & 50,000 & 9,000 & 1,000 & 60,000 & 3,900 & 100 & 4,000 & 64,000 \\
\hline $30-35$ & 26,000 & 10,000 & 2,000 & 38,000 & 2,700 & 300 & 3,000 & 41,000 \\
\hline $35-40$ & 14,000 & 10,000 & 3,000 & $\mathbf{2 7}, 000$ & 2,400 & 600 & 3,000 & 30,000 \\
\hline $40-45$ & 9,000 & 8,000 & 4,000 & 21,000 & 2,000 & 1,000 & $\mathbf{3}, 000$ & 24,000 \\
\hline $45-50$ & 6,000 & 6,000 & 5,000 & 17,000 & 500 & 500 & 1,000 & 18,000 \\
\hline $50-55$ & 4,000 & 4,000 & 6,000 & 14,000 & 400 & 600 & 1,000 & 15,000 \\
\hline $55-60$ & 2,000 & 3,000 & 6,000 & 11,000 & 200 & 500 & 700 & 11,700 \\
\hline \multirow[t]{2}{*}{$60-65$} & 1,000 & 2,000 & 5,000 & 8,000 & 100 & 200 & 300 & 8,300 \\
\hline & 316,000 & 58,000 & 32,000 & 406,000 & 21,200 & 3,800 & 25,000 & 431,000 \\
\hline
\end{tabular}


TABLE IV.

Estimated Number of Persons becoming Members of Approved Societies as at 1 May 1912.

IRELAND.

Men.

\begin{tabular}{|c|c|c|c|}
\hline Ages & Uompulsory & Voluntary & Both Classes \\
\hline $\begin{array}{l}16-20 \\
20-25 \\
25-30 \\
30-35 \\
35-40 \\
40-45 \\
45-50 \\
50-55 \\
55-60 \\
60-65\end{array}$ & $\begin{array}{l}70,000 \\
77,000 \\
65,000 \\
62,000 \\
54,000 \\
46,000 \\
40,000 \\
32,000 \\
24,000 \\
19,000\end{array}$ & $\begin{array}{r}15,000 \\
22,000 \\
24,000 \\
27,000 \\
29,000 \\
30,000 \\
14,000 \\
11,000 \\
8,000 \\
5,000\end{array}$ & $\begin{array}{l}85,000 \\
99,000 \\
89,000 \\
89,000 \\
83,000 \\
76,000 \\
54,000 \\
43,000 \\
32,000 \\
24,000\end{array}$ \\
\hline & 489,000 & 185,000 & 674,000 \\
\hline
\end{tabular}

Women.

\begin{tabular}{|c|c|c|c|c|c|c|c|c|}
\hline \multirow{2}{*}{ Ages } & \multicolumn{4}{|c|}{ Compulsory } & \multicolumn{3}{|c|}{ Voluntary } & \multirow{2}{*}{$\begin{array}{c}\text { Both } \\
\text { Classes }\end{array}$} \\
\hline & Spinsters & Married & Widows & Total & Spinsters & Widows & Total & \\
\hline $15-20$ & 54,000 & & $\ldots$ & 54,000 & 2,000 & $\ldots$ & 2,000 & 56,000 \\
\hline $20-25$ & 62,000 & 3,000 & & 65,000 & 3,000 & $\ldots$ & 3,000 & 68,000 \\
\hline $25-30$ & 35,000 & 6,000 & 1,000 & 42,000 & 1,900 & 100 & 2,000 & 44,000 \\
\hline $30-35$ & 21,000 & 8,000 & 2,000 & 31,000 & $\mathbf{2 , 7 0 0}$ & 300 & 3,000 & 34,000 \\
\hline $35-40$ & 13,000 & 8,000 & 3,000 & 24,000 & 3,200 & 800 & 4,000 & 28,000 \\
\hline $40-45$ & 8,000 & 7,000 & 4,000 & 19,000 & $\mathbf{3 , 3 0 0}$ & 1,700 & 5,000 & $\mathbf{2 4 , 0 0 0}$ \\
\hline $45-50$ & 5,000 & 5,000 & 5,000 & 15,000 & 1,600 & 1,400 & 3,000 & 18,000 \\
\hline $50-55$ & 4,000 & 4,000 & 5,000 & 13,000 & 1,200 & 1,800 & 3,000 & 16,000 \\
\hline $55-60$ & 2,000 & 3,000 & 6,000 & 11,000 & 600 & 1,400 & 2,000 & 13,000 \\
\hline \multirow[t]{2}{*}{$60-65$} & 1,000 & 2,000 & 5,000 & 8,000 & 200 & 800 & 1,000 & 9,000 \\
\hline & 205,000 & 46,000 & 31,000 & 282,000 & 19,700 & 8,300 & 28,000 & 310,000 \\
\hline
\end{tabular}


TABLE V.

Estimated Number of Persons becoming Members of Approved Societies as at 1 May 1912.

UNIJ'ED KingdoM.

Men.

\begin{tabular}{|c|c|c|c|}
\hline Ages & Compulsory & VoIuntary & Both Classes \\
\hline $\begin{array}{l}16-20 \\
20-25 \\
25-30 \\
30-35 \\
35-40 \\
40-45 \\
45-50 \\
50-55 \\
55-60 \\
60-65\end{array}$ & $\begin{array}{r}1,192,000 \\
1,340,000 \\
1,211,000 \\
1,109,000 \\
981,000 \\
839,000 \\
694,000 \\
\mathbf{5 4 3 , 0 0 0} \\
\mathbf{3 9 8 , 0 0 0} \\
\mathbf{2 7 2 , 0 0 0}\end{array}$ & $\begin{array}{r}29,000 \\
55,000 \\
76,000 \\
95,000 \\
110,000 \\
118,000 \\
56,000 \\
41,000 \\
28,000 \\
17,000\end{array}$ & $\begin{array}{r}1,221,000 \\
1,395,000 \\
1,287,000 \\
1,204,000 \\
1,091,000 \\
957,000 \\
750,000 \\
584,000 \\
426,000 \\
289,000\end{array}$ \\
\hline & $8,579,000$ & 625,000 & $9,201,000$ \\
\hline
\end{tabular}

Women.

\begin{tabular}{|c|c|c|c|c|c|c|c|c|}
\hline \multirow{2}{*}{ Ages } & \multicolumn{4}{|c|}{ COMPJLSORY } & \multicolumn{3}{|c|}{ VOLUNTARY } & \multirow{2}{*}{$\begin{array}{c}\text { Both } \\
\text { Classes }\end{array}$} \\
\hline & Spinsters & Married & Widows & Total & Spinsters & Widows & Total & \\
\hline $16-20$ & 891,000 & 7,000 & & 898,000 & 26,000 & & 26,000 & 924,000 \\
\hline $20-25$ & $821,0(10$ & 44,000 & 2,000 & 867,000 & 31,900 & 100 & 32,000 & 899,000 \\
\hline $25-30$ & 433,000 & 76,000 & 10,000 & 519,000 & 25,300 & 700 & 26,000 & 545,000 \\
\hline $30-35$ & 237,000 & 94,000 & 22,000 & 353,000 & 22,800 & 2,200 & $\mathbf{2 5}, 000$ & 378,000 \\
\hline $35-40$ & 141,000 & 93,000 & 34,000 & 268,000 & 20,900 & 5,100 & 26,000 & 294,000 \\
\hline $40-4 \overline{5}$ & 91,000 & 79,000 & 46,000 & 216,000 & 18,600 & 9,400 & 28,000 & $\mathbf{2 4 , 4 , 0 0 0}$ \\
\hline $45-50$ & 60,000 & 62,000 & 54,000 & 176,000 & 7,300 & 6,700 & 14,000 & 190,000 \\
\hline $50-55$ & 40,000 & 45,000 & 58,000 & 143,000 & 5.200 & 7,800 & 13,000 & 156,000 \\
\hline $55-60$ & 23,000 & 30,000 & 55,000 & 108,000 & 2,600 & 6,100 & 8,700 & 116,700 \\
\hline \multirow[t]{2}{*}{$60-65$} & 14,000 & 18,000 & 48,000 & 80,000 & 1,200 & 4,100 & 5,300 & 85,300 \\
\hline & $2,751,000$ & 548,000 & 329,000 & $3,628,000$ & 161,800 & 42,200 & 204,000 & $3,832,000$ \\
\hline
\end{tabular}




\author{
TABLE VI.
}

Estimated Number of Members of Approved Societies (Contributors under age 70$)$.

ENGLAND.

\begin{tabular}{|c|c|c|c|c|c|c|}
\hline \multirow{2}{*}{ Year } & \multicolumn{3}{|c|}{ COMPULSORY } & \multicolumn{3}{|c|}{ Voluntary } \\
\hline & Men & Women & Both Sexes & Men & Women & Both Sexes \\
\hline $\begin{array}{l}1912-13 \\
1917-18 \\
1922-23 \\
1927-28 \\
1932-33\end{array}$ & $\begin{array}{l}6,682,000 \\
7,468,000 \\
8,118,000 \\
8,756,000 \\
9,373,000\end{array}$ & $\begin{array}{l}\mathbf{2 , 7 8 2 , 0 0 0} \\
3,083,000 \\
\mathbf{3 , 3 4 7 , 0 0 0} \\
\mathbf{3 , 6 1 0 , 0 0 0} \\
3,873,000\end{array}$ & $\begin{array}{r}9,464,000 \\
10,551,000 \\
11,465,000 \\
12,366,000 \\
13,246,000\end{array}$ & $\begin{array}{l}375,000 \\
376,000 \\
362,000 \\
\mathbf{3 4 6 , 0 0 0} \\
\mathbf{3 2 8 , 0 0 0}\end{array}$ & $\begin{array}{l}143,000 \\
139,000 \\
137,000 \\
135,000 \\
134,000\end{array}$ & $\begin{array}{l}518,000 \\
515,000 \\
499,000 \\
481,000 \\
462,000\end{array}$ \\
\hline \multicolumn{7}{|c|}{ WALES. } \\
\hline $\begin{array}{l}1912-13 \\
1917-18 \\
1922-23 \\
1927-28 \\
1932-33\end{array}$ & $\begin{array}{l}419,000 \\
468,000 \\
509,000 \\
549,000 \\
588,000\end{array}$ & $\begin{array}{l}158,000 \\
175,000 \\
192,000 \\
206,000 \\
220,000\end{array}$ & $\begin{array}{l}\mathbf{5 7 7}, 000 \\
643,000 \\
701,000 \\
755,000 \\
808,000\end{array}$ & $\begin{array}{l}23,000 \\
24,000 \\
23,000 \\
22,000 \\
21,000\end{array}$ & $\begin{array}{l}8,000 \\
8,000 \\
7,000 \\
7,000 \\
7,000\end{array}$ & $\begin{array}{l}31,000 \\
32,000 \\
30,000 \\
29,000 \\
28,000\end{array}$ \\
\hline \multicolumn{7}{|c|}{ SCOTJAND. } \\
\hline $\begin{array}{l}1912-13 \\
1917-18 \\
1922-23 \\
1927-28 \\
1932-33\end{array}$ & $\begin{array}{r}989,000 \\
1,102,000 \\
1,197,000 \\
\mathbf{1}, \mathbf{2 8 4 , 0 0 0} \\
\mathbf{1 , 3 7 2 , 0 0 0}\end{array}$ & $\begin{array}{l}406,000 \\
449,000 \\
482,000 \\
518,000 \\
553,000\end{array}$ & $\begin{array}{l}1,395,000 \\
1,551,000 \\
1,679,000 \\
1,802,000 \\
1,925,000\end{array}$ & $\begin{array}{l}42,000 \\
41,000 \\
40,000 \\
39,000 \\
34,000\end{array}$ & $\begin{array}{l}25,000 \\
23,000 \\
23,000 \\
23,000 \\
23,000\end{array}$ & $\begin{array}{l}67,000 \\
64,000 \\
63,000 \\
62,000 \\
57,000\end{array}$ \\
\hline \multicolumn{7}{|c|}{ IRELAND. } \\
\hline $\begin{array}{l}1912-13 \\
1917-18 \\
1922-23 \\
1927-28 \\
1932-33\end{array}$ & $\begin{array}{l}489,000 \\
548,000 \\
588,000 \\
622,000 \\
651,000\end{array}$ & $\begin{array}{l}\mathbf{2 8 2}, 000 \\
313,000 \\
\mathbf{3 3 4}, 000 \\
\mathbf{3 5 3}, 000 \\
\mathbf{3 6 9}, 000\end{array}$ & $\begin{array}{r}771,000 \\
861,000 \\
922,000 \\
975,000 \\
1,020,000\end{array}$ & $\begin{array}{l}185,000 \\
179,000 \\
174,000 \\
166,000 \\
157,000\end{array}$ & $\begin{array}{l}28,000 \\
28,000 \\
29,000 \\
27,000 \\
26,000\end{array}$ & $\begin{array}{l}213,000 \\
207,000 \\
203,000 \\
193,000 \\
183,000\end{array}$ \\
\hline \multicolumn{7}{|c|}{ UNITED KINGDOM. } \\
\hline $\begin{array}{l}1912-13 \\
1917-18 \\
1922-23 \\
1927-28 \\
1932-33\end{array}$ & $\begin{array}{r}8,579,000 \\
9,586,000 \\
10,412,000 \\
11,211,000 \\
11,984,000\end{array}$ & $\begin{array}{l}3,628,000 \\
4,020,000 \\
4,355,000 \\
\mathbf{4 , 6 8 7 , 0 0 0} \\
5,015,000\end{array}$ & $\begin{array}{l}12,207,000 \\
13,606,000 \\
14,767,000 \\
15,898,000 \\
16,999,000\end{array}$ & $\begin{array}{l}625,000 \\
620,000 \\
599,000 \\
573,000 \\
510,000\end{array}$ & $\begin{array}{l}204,000 \\
198,000 \\
196,000 \\
192,000 \\
190,000\end{array}$ & $\begin{array}{l}829,000 \\
818,000 \\
795,000 \\
765,000 \\
730,000\end{array}$ \\
\hline
\end{tabular}


TABLE VII.

The Aggregate Contributions to be Received from Employers and Insured Persons, i.e., Members of Approved Societies, in the undermentioned years.

ENGLAND.

\begin{tabular}{|c|c|c|c|c|c|c|c|}
\hline \multirow{2}{*}{ Year } & \multicolumn{3}{|c|}{ COMPULSORY } & \multicolumn{3}{|c|}{ VOLUNTARY } & \multirow{2}{*}{$\begin{array}{c}\text { Total, } \\
\text { Both Classes }\end{array}$} \\
\hline & Men & Women & Both Sexes & Men & Women & Both Sexes & \\
\hline $1912-13$ & $\frac{\mathfrak{E}}{7,097,000}$ & $\begin{array}{c}\mathcal{E} \\
2,543,000\end{array}$ & $\stackrel{ \pm}{9,6 \pm 0,000}$ & $\begin{array}{c}\dot{E} \\
496,000\end{array}$ & $\frac{\dot{E}}{162,400}$ & $\frac{£}{658,400}$ & $\begin{array}{c}\dot{E} \\
10,298,400\end{array}$ \\
\hline $1913-14$ & $9,672,000$ & $3,460,000$ & $13,132,000$ & 657,000 & 214,300 & 871,300 & $14,003,300$ \\
\hline 19$] 4-15$ & $9,880,000$ & $3,531,000$ & $13,411,000$ & 653,000 & 212,300 & 865,300 & $14,276,300$ \\
\hline $1915-16$ & $10,090,000$ & $3,600,000$ & $13,690,000$ & 649,000 & 210,400 & 859,400 & $14,549,400$ \\
\hline $1916-17$ & $10,299,000$ & $3,670,000$ & $13,969, \mathrm{COO}$ & 641,000 & 208,600 & 849,600 & $14,818,600$ \\
\hline $1917-18$ & $10,494,000$ & $3,738,000$ & $14,232,000$ & 633,000 & 206,900 & 839,900 & $15,071,900$ \\
\hline $1922-23$ & $11,384,000$ & $4,055,000$ & $15,439,000$ & 570,000 & 190,200 & 760,200 & $16,199,200$ \\
\hline $1927-28$ & $12,254,000$ & $4,363,000$ & $16,617,000$ & 514,000 & 175,400 & 689,400 & $17,306,400$ \\
\hline 1932-33 & $13,101,000$ & $4,673,000$ & $17,774,000$ & 453,000 & 166,400 & 619,400 & $18,393,400$ \\
\hline
\end{tabular}

WALES.

\begin{tabular}{|r|r|r|r|r|r|r|r}
\hline $1912-13$ & 445,000 & 145,000 & 590,000 & 31,000 & 9,300 & 40,300 & 630,300 \\
$1913-14$ & 606,000 & 198,000 & 804,000 & 41,000 & 12,300 & 53,300 & 857,300 \\
$1914-15$ & 620,000 & 202,000 & 822,000 & 41,000 & 12,100 & 53,100 & 875,100 \\
$1915-16$ & 632,000 & 206,000 & 838,000 & 40,000 & 12,000 & 52,000 & 890,000 \\
$1916-17$ & 646,000 & 209,000 & 855,000 & 40,000 & 11,900 & 51,900 & 906,900 \\
$1917-18$ & 658,000 & 213,000 & 871,000 & 40,000 & 11,800 & 51,800 & 922,800 \\
$1922-23$ & 714,000 & 231,000 & 945,000 & 36,000 & 10,900 & 46,900 & 991,900 \\
$1927-28$ & 768,000 & 249,000 & $1,017,000$ & 32,000 & 10,000 & 42,000 & $1,059,000$ \\
$1932-33$ & 821,000 & 266,000 & $1,087,000$ & 28,000 & 9,500 & 37,500 & $1,124,500$ \\
\hline
\end{tabular}

\begin{tabular}{|c|c|c|c|c|c|c|c|}
\hline \multicolumn{8}{|c|}{ SCOTLAND. } \\
\hline $1912-13$ & $1,050,000$ & 371,000 & $1,421,000$ & 56,000 & 28,100 & 84,100 & $1,505,100$ \\
\hline & 1,000 & 000 & 7,000 & 74,000 & 37,400 & 111 & 400 \\
\hline $1914-15$ & $1,461,000$ & 516,000 & 977,000 & 73,000 & 37,400 & 110,400 & $2,087,400$ \\
\hline $1915-16$ & $1,492,000$ & 526,000 & 18,000 & 72,000 & 37,500 & 109,500 & $2,127,500$ \\
\hline $1916-17$ & $1,522,000$ & 536,000 & 058,000 & 71,000 & 37,300 & 108,300 & $2,166,300$ \\
\hline $1917-18$ & $1,551,000$ & 546,000 & $, 097,000$ & 69,000 & 37,400 & 106,400 & $2,203,400$ \\
\hline $1922-23$ & 9,000 & 583 & 2,000 & & 34 & & $2,357,700$ \\
\hline $1927-28$ & $1,799,000$ & 628,006 & $2,427,000$ & 56 , & 32,300 & 88,300 & $2,515,300$ \\
\hline $1932-33$ &, 000 & 671 & $2,590,000$ & 47,000 & 31,400 & 78,400 &, 400 \\
\hline \multicolumn{8}{|c|}{ IRELAND. } \\
\hline 191 & 00 & 193 & 603,000 & 198,000 & 29 & 227,800 & 800 \\
\hline $1913-14$ & 557,000 & 263,000 & 820,000 & 261,000 & 39,400 & 300,400 & $1,120,400$ \\
\hline $1914-15$ & 568,000 & 269,000 & 837,000 & 258,000 & 39,300 & 297,300 & $1,134,300$ \\
\hline $1915-16$ & 581,000 & 275,000 & 856,000 & 100 & 39 & 294,000 & 0,000 \\
\hline $1916-17$ & 593,000 & 280,000 & 873,000 & 253,000 & 38,700 & 291,700 & $1,164,700$ \\
\hline $1917-18$ & 604,000 & 284,000 & 888,000 & 251,000 & 38,500 & 289,500 & $1,177,500$ \\
\hline $1922-23$ & 644,000 & 303,000 & 947,000 & 226,000 & 33,500 & 259,500 & $1,206,500$ \\
\hline $1927-28$ & 680,000 & 318,000 & 998,000 & 199,000 & 28,700 & 227,700 & $1,225,700$ \\
\hline $1932-33$ & 710,000 & 330,000 & $1,040,000$ & 175,000 & 24,800 & 199,800 & $1,239,800$ \\
\hline
\end{tabular}

UNITED KingDOM.

\begin{tabular}{|c|c|c|c|c|c|c|c|}
\hline $1912-13$ & $9,002,000$ & $3,252,000$ & $12,254,000$ & 781,000 & 229,600 & $1,010,600$ & $13,264,600$ \\
\hline $1913-14$ & $12,266,000$ & $4,427,000$ & $16,693,000$ & $1,033,000$ & 303,400 & $1,336,400$ & $18,029,400$ \\
\hline $1914-15$ & $12,529,000$ & $4,518,000$ & $17,047,000$ & $1,025,000$ & 301,100 & $1,326,100$ & $18,373,100$ \\
\hline $1915-16$ & $12,795,000$ & $4,607,000$ & $17,402,000$ & $1,016,000$ & 298,900 & $1,314,900$ & $18,716,900$ \\
\hline 1916-17 & $13,060,000$ & $4,695,000$ & $17,755,000$ & $1,005,000$ & 296,500 & $1,301,500$ &, 500 \\
\hline 1917-18 & $13,307,000$ & $4,781,000$ & $18,088,000$ & 993,000 & 294,600 & $1,287,600$ & $19,375,600$ \\
\hline 1922-23 & $14,421,000$ & $5,172,000$ & $19,593,000$ & 893,000 & 269,300 & $1,162,300$ & $20,755,300$ \\
\hline & & $5,558,000$ & 21, &, 000 & 246,400 & $1,047,400$ & 6,400 \\
\hline 1932-33 & $16,551,000$ & $5,940,000$ & $\mathbf{2 2}, \mathbf{4 9 1 , 0 0 0}$ & 703,000 & 232,100 & 935,100 & $23,426,100$ \\
\hline
\end{tabular}




\section{TABLE VIII.}

Total Amount required in each of the under-mentioned financial years to meet the Cost of the various "Minimum Benefits."

ENGLAND.

Men (Compulsory).

\begin{tabular}{|c|c|c|c|c|c|c|c|c|}
\hline \multirow{2}{*}{ Year } & \multicolumn{5}{|c|}{ Benefits } & \multirow{2}{*}{$\underset{\text { Bunefits }}{\text { Total }}$} & \multirow{2}{*}{$\begin{array}{l}\text { Cost of } \\
\text { Admmins- } \\
\text { tration }\end{array}$} & \multirow{2}{*}{$\begin{array}{l}\text { Total } \\
\text { Outgo }\end{array}$} \\
\hline & $a$ & $b$ & $c$ & $d$ & $e$ & & & \\
\hline $\begin{array}{l}1912-13 \\
1913-1.4 \\
1914-15 \\
1915-16 \\
1916-17 \\
1917-18 \\
1922-23 \\
1927-28 \\
1932-33\end{array}$ & $\begin{array}{c}£ \\
507,000 \\
2,075,000 \\
2,123,000 \\
2,170,000 \\
2,217,000 \\
2,264,000 \\
3,492,000 \\
2,708,000 \\
2,913,000\end{array}$ & $\begin{array}{c}£ \\
317,000 \\
432,000 \\
442,000 \\
452,000 \\
462,000 \\
471,000 \\
519,000 \\
565,000 \\
607,000\end{array}$ & \begin{tabular}{|c|}
$£$ \\
751,000 \\
$3,119,000$ \\
$3,233,000$ \\
$3,348,000$ \\
$3,463,000$ \\
$3,635,000$ \\
$4,285,000$ \\
$4,629,000$ \\
$5,001,000$
\end{tabular} & $\begin{array}{c}£ \\
\ldots \\
\ldots \\
852,000 \\
1,228,000 \\
1,320,000 \\
1,388,000 \\
1,601,000 \\
1,810,000 \\
2,007,000\end{array}$ & $\begin{array}{c}\mathfrak{E} \\
231,000 \\
940,000 \\
954,000 \\
968,000 \\
933,000 \\
997,000 \\
1,076,000 \\
1,146,000 \\
1,222,000\end{array}$ & \begin{tabular}{c}
\multirow{\ddagger}{\pm}{} \\
$1,806,000$ \\
$6,566,000$ \\
$7,604,000$ \\
$8,166,000$ \\
$8,445,000$ \\
$8,755,000$ \\
$9,973,000$ \\
$10,858,000$ \\
$11,750,000$
\end{tabular} & $\begin{array}{c}\mathfrak{E} \\
933,000 \\
1,271,000 \\
1,299,000 \\
1,326,(100 \\
1,353,000 \\
1,380,000 \\
1,496,000 \\
1,610,000 \\
1,721,000\end{array}$ & $\begin{array}{r}2,739,000 \\
7,837,000 \\
8,903,000 \\
9,492,000 \\
9,798,000 \\
10,135,000 \\
11,469,000 \\
12,468,000 \\
13,471,000\end{array}$ \\
\hline \multicolumn{9}{|c|}{ Men (Voluntary). } \\
\hline $\begin{array}{l}1912-13 \\
1913-14 \\
1914-15 \\
1915-16 \\
1916-17 \\
1917-18 \\
1922-23 \\
1927-28 \\
1932-33\end{array}$ & $\begin{array}{r}27,200 \\
112,900 \\
112,900 \\
112,900 \\
112,90) \\
112,900 \\
110,100 \\
106,300 \\
98,800\end{array}$ & $\begin{array}{l}17,900 \\
23,500 \\
23,500 \\
23,500 \\
23,500 \\
23,500 \\
22,600 \\
21,700 \\
20,500\end{array}$ & $\begin{array}{r}48,900 \\
201,400 \\
205,100 \\
208,900 \\
212,700 \\
215,500 \\
223,000 \\
228,700 \\
229,600\end{array}$ & $\begin{array}{c}\ldots \\
\ddot{2}, 700 \\
76,300 \\
81,900 \\
85,700 \\
103,600 \\
121,400 \\
140,200\end{array}$ & $\begin{array}{l}42,300 \\
55,500 \\
53,600 \\
51,800 \\
50,800 \\
41,400 \\
33900 \\
30,100\end{array}$ & $\begin{array}{r}94,000 \\
380,100 \\
449,700 \\
475,200 \\
482,800 \\
\mathbf{4 8 9 , 4 0 0} \\
500,700 \\
512,000 \\
519,200\end{array}$ & $\begin{array}{l}51,800 \\
68,700 \\
68,700 \\
68,700 \\
68,700 \\
67,800 \\
64,900 \\
62,100 \\
58,300\end{array}$ & $\begin{array}{l}\mathbf{1 4 5 , 8 0 0} \\
448,800 \\
518,400 \\
\mathbf{5 4 3 , 9 0 0} \\
\mathbf{5 5 1 , 5 0 0} \\
\mathbf{5 5 6 , 2 0 0} \\
\mathbf{5 6 5 , 6 0 0} \\
\mathbf{5 7 4}, 100 \\
\mathbf{5 7 7 , 5 0 0}\end{array}$ \\
\hline \multicolumn{9}{|c|}{ Women (Compulsory). } \\
\hline $\begin{array}{l}1912-13 \\
1913-14 \\
1914-15 \\
1915-16 \\
1916-17 \\
1917-18 \\
1922-23 \\
1927-28 \\
1932-33\end{array}$ & $\begin{array}{r}211,000 \\
863,000 \\
881,000 \\
898,000 \\
916,000 \\
935,000 \\
1,024,000 \\
1,109,000 \\
1,195,000\end{array}$ & $\begin{array}{l}133,000 \\
179,000 \\
183,000 \\
185,000 \\
190,000 \\
194,000 \\
213,000 \\
231,000 \\
249,000\end{array}$ & $\begin{array}{r}222,000 \\
917,000 \\
946,000 \\
974,000 \\
998,000 \\
1,029,000 \\
1,167,000 \\
1,274,000 \\
1,393,000\end{array}$ & $\begin{array}{c}\ldots \\
\ldots \\
239,000 \\
346,000 \\
370,000 \\
392,000 \\
448,000 \\
514,000 \\
579,000\end{array}$ & $\begin{array}{r}30,000 \\
124,000 \\
127,000 \\
129,000 \\
131,000 \\
133,000 \\
140,000 \\
150,000 \\
161,000\end{array}$ & $\begin{array}{r}596,000 \\
2,083,000 \\
2,376,000 \\
2,532,000 \\
2,605,000 \\
2,633,000 \\
2,992,000 \\
3,278,000 \\
\mathbf{3}, \mathbf{5 7 7}, 000\end{array}$ & $\begin{array}{l}390,000 \\
531,000 \\
542,000 \\
552,000 \\
563,000 \\
573,000 \\
622,000 \\
669,000 \\
717,000\end{array}$ & $\begin{array}{r}986,000 \\
2,614,000 \\
2,918,000 \\
3,084,000 \\
3,168,000 \\
3,256,000 \\
3,614,000 \\
3,947,000 \\
4,294,000\end{array}$ \\
\hline \multicolumn{9}{|c|}{ Women (Voluntary). } \\
\hline $\begin{array}{l}1912-13 \\
1913-14 \\
1914-15 \\
1915-16 \\
1916-17 \\
1917-18 \\
1922-23 \\
1927-28 \\
1932-33\end{array}$ & $\begin{array}{l}10,700 \\
42,800 \\
42,700 \\
42,600 \\
42,500 \\
42,400 \\
42,500 \\
42,400 \\
42,800\end{array}$ & $\begin{array}{l}6,600 \\
8,900 \\
8,900 \\
8,900 \\
8,900 \\
8,900 \\
8,800 \\
8,800 \\
8,900\end{array}$ & $\begin{array}{l}13,000 \\
52,100 \\
52,500 \\
53,000 \\
53,400 \\
53,800 \\
57,100 \\
58,400 \\
59,000\end{array}$ & $\begin{array}{c}\ldots \\
\ldots \\
17,000 \\
24,500 \\
26,100 \\
27,700 \\
31,300 \\
34,200 \\
38,200\end{array}$ & $\begin{array}{l}\cdots \\
\cdots \\
\cdots \\
\cdots \\
\cdots \\
\cdots \\
\cdots \\
\cdots \\
\cdots\end{array}$ & $\begin{array}{r}30,300 \\
103,800 \\
121,100 \\
129,000 \\
130,900 \\
132,800 \\
139,700 \\
143,800 \\
148,900\end{array}$ & $\begin{array}{l}19,600 \\
26,000 \\
26,000 \\
25,900 \\
25,800 \\
25,700 \\
25,400 \\
25,000 \\
24,700\end{array}$ & $\begin{array}{r}49,900 \\
129,800 \\
147,100 \\
154,900 \\
156,700 \\
158,500 \\
165,100 \\
168,000 \\
173,600\end{array}$ \\
\hline
\end{tabular}


TABLE IX.

Total Amount required in each of the undermentioned financial years to meet the Cost of the various "Minimum Benefits."

WALES.

Men (Compulsory).

\begin{tabular}{|c|c|c|c|c|c|c|c|c|}
\hline \multirow[b]{2}{*}{ Year } & \multicolumn{5}{|c|}{ BENEFITS } & \multirow{2}{*}{$\begin{array}{c}\text { Total } \\
\text { Minimum } \\
\text { Benefits }\end{array}$} & \multirow{2}{*}{$\begin{array}{c}\text { Cost of } \\
\text { Admunis- } \\
\text { tration }\end{array}$} & \multirow{2}{*}{$\begin{array}{l}\text { Total } \\
\text { Outgo }\end{array}$} \\
\hline & $a$ & $b$ & $c$ & $d$ & $e$ & & & \\
\hline & $\mathfrak{E}^{2}$ & $\alpha$ & $£$ & E & $£$ & \pm & $f$ & $\mathscr{E}$ \\
\hline $1912-13$ & 32,000 & 13,000 & 47,000 & & 15,000 & 107,000 & 58,000 & 165,000 \\
\hline $1913-14$ & 130,000 & 27,000 & 195,000 & & 59,000 & 411,000 & 80,000 & 491,900 \\
\hline $1914-15$ & 133,000 & 28,000 & 203,000 & 53,000 & 60 & 47 & 81,000 & 558,000 \\
\hline $1915-16$ & 136,000 & 28,000 & 210,000 & 77,000 & 61,000 & 512,000 & 83,000 & 595,000 \\
\hline $1916-17$ & 139,000 & 29,000 & 217,000 & 83,000 & 61,000 & 529,000 & 85,000 & 614,000 \\
\hline $1917-18$ & 142,000 & 30,000 & 228,000 & 87,0 & 62,0 & 549, & 86,000 & 635,000 \\
\hline $1922-23$ & 156,000 & 33,000 & 269,000 & 100,000 & 67,000 & 625,000 & 91,000 & 719,000 \\
\hline $1927-28$ & 170,000 & 35,000 & 290,000 & 113,000 & 72,000 & 680,000 & 101,000 & 781,000 \\
\hline 1932-33 & 183,000 & 38,000 & 314,000 & 126,000 & 77,000 & 738,000 & 108,000 & 846,000 \\
\hline
\end{tabular}

Men (Voluntary).

\begin{tabular}{r|r|r|r|r|r|r|r|r}
\hline $1912-13$ & 1,800 & 1,100 & 3,100 & $\ldots$ & $\ldots$ & 6,000 & 3,200 & 9,200 \\
$1913-14$ & 7,100 & 1,500 & 12,600 & $\ldots$ & 2,700 & 23,900 & 4,300 & 28,200 \\
$1914-15$ & 7,100 & 1,500 & 12,900 & 3,300 & 3,500 & 28,300 & 4,300 & 32,600 \\
$1915-16$ & 7,100 & 1,500 & 13,100 & 4,700 & 3,400 & 29,800 & 4,300 & 34,100 \\
$1916-17$ & 7,100 & 1,500 & 13,300 & 5,100 & 3,200 & 30,200 & 4,300 & 34,500 \\
$1917-18$ & 7,100 & $\mathbf{1 , 5 0 0}$ & 13,500 & 5,300 & 3,200 & 30,600 & 4,200 & 34,800 \\
$1922-23$ & $\mathbf{6 , 9 0 0}$ & $\mathbf{1}, 400$ & 14,000 & $\mathbf{6 , 4 0 0}$ & 2,600 & 31,300 & 4,100 & $\mathbf{3 5}, 400$ \\
$1927-28$ & 6,700 & $\mathbf{1 , 3 0 0}$ & 14,300 & $\mathbf{7 , 6 0 0}$ & $\mathbf{2 , 1 0 0}$ & 32,000 & 3,900 & 35,900 \\
$1932-33$ & $\mathbf{6 , 2 0 0}$ & $\mathbf{1 , 2 0 0}$ & $\mathbf{1 4 , 4 0 0}$ & $\mathbf{8 , 8 0 0}$ & $\mathbf{1 , 9 0 0}$ & 32,500 & $\mathbf{3 , 7 0 0}$ & $\mathbf{3 6 , 2 0 0}$ \\
& & & & & & & & \\
\hline
\end{tabular}

Women (Compulsory).

\begin{tabular}{r|r|r|r|r|r|r|r|r}
\hline $1912-13$ & 12,000 & 8,000 & 13,000 & $\ldots$ & 2,000 & 35,000 & 22,000 & 57,000 \\
$1913-14$ & 49,000 & 10,000 & 52,000 & $\ldots$ & 7,000 & 118,000 & 30,000 & 148,000 \\
$1914-15$ & 50,000 & 10,000 & 54,000 & 14,000 & 7,000 & 135,000 & 31,000 & 166,000 \\
$1915-16$ & 51,000 & 11,000 & 56,000 & 20,000 & 7,000 & 145,000 & 32,000 & 177,000 \\
$1916-17$ & 52,000 & 11,000 & 57,000 & 21,000 & 8,000 & 149,000 & 32,000 & 181,000 \\
$1917-18$ & 52,000 & 11,000 & 59,000 & 22,000 & 8,000 & 152,000 & 33,000 & 185,000 \\
$1922-23$ & 58,000 & 12,000 & 67,000 & 26,000 & 8,000 & 171,000 & 35,000 & 206,000 \\
$1927-23$ & 63,000 & 13,000 & 73,000 & 29,000 & 9,000 & 187,000 & 38,000 & 225,000 \\
$1932-33$ & 68,000 & 14,000 & 80,000 & 33,000 & 9,000 & 201,000 & 41,000 & 245,000 \\
& & & & & & & & \\
\hline
\end{tabular}

Women (Voluntary).

\begin{tabular}{|c|c|c|c|c|c|c|c|c|}
\hline $1912-13$ & 600 & 400 & 700 & $\ldots$ & $\ldots$ & 1,700 & 1,100 & 2,800 \\
\hline $1913-14$ & 2,400 & 500 & 3,000 & & $\ldots$ & 5,900 & 1,500 & 7,400 \\
\hline 1914-15 & 2,400 & 500 & 3,000 & 1,000 & $\ldots$ & 6,900 & 1,500 & 8,400 \\
\hline $1915-16$ & 2,400 & 500 & 3,000 & 1,400 & $\ldots$ & 7,300 & 1,500 & 8,800 \\
\hline $1916-17$ & $\mathbf{2 , 4 0 0}$ & 500 & 3,100 & 1,500 & $\ldots$ & 7,500 & 1,500 & 9,000 \\
\hline $1917-18$ & 2,400 & 500 & 3,100 & 1,600 & • & 7,600 & 1,500 & 9,100 \\
\hline $1922-23$ & 2,400 & 500 & 3,300 & 1,800 & $\ldots$ & 8,000 & 1,400 & 9,400 \\
\hline $1927-28$ & 2,400 & 500 & 3,300 & 2,000 & $\ldots$ & 8,200 & 1,400 & 9,600 \\
\hline $1932-33$ & $\mathbf{2 , 4 0 0}$ & 500 & 3,400 & 2,200 & $\ldots$ & 8,500 & 1,400 & 9,900 \\
\hline
\end{tabular}


TABLE X.

Total Amount required in each of the undermentioned financial years to meet the Cost of the various "Minimum Benefits."

SCOTLAND.

Men (Compulsory.)

\begin{tabular}{|c|c|c|c|c|c|c|c|c|}
\hline \multirow{2}{*}{ Year } & \multicolumn{5}{|c|}{ Benefits } & \multirow{2}{*}{$\begin{array}{c}\text { Total } \\
\text { Minimum } \\
\text { Benefits }\end{array}$} & \multirow{2}{*}{$\begin{array}{c}\text { Cost of } \\
\text { Adminis- } \\
\text { tration }\end{array}$} & \multirow{2}{*}{$\begin{array}{l}\text { Total } \\
\text { Outgo }\end{array}$} \\
\hline & $a$ & $b$ & $c$ & $d$ & $e$ & & & \\
\hline $\begin{array}{l}1912-13 \\
1913-14 \\
1914-15 \\
1915-16 \\
1916-17 \\
1917-18 \\
1922-23 \\
1927-28 \\
1932-33\end{array}$ & $\begin{array}{c}£ \\
75,000 \\
308,000 \\
314,000 \\
321,000 \\
328,000 \\
334,000 \\
367,000 \\
396,000 \\
425,000\end{array}$ & $\begin{array}{c}\boldsymbol{E} \\
\mathbf{4 6 , 0 0 0} \\
\mathbf{6 4 , 0 0 0} \\
\mathbf{6 5}, 000 \\
67,000 \\
68,000 \\
70,000 \\
77,000 \\
83,000 \\
89,000\end{array}$ & $\begin{array}{c}£ \\
109,000 \\
454,000 \\
471,000 \\
488,000 \\
505,000 \\
524,000 \\
614,000 \\
666,000 \\
718,000\end{array}$ & $\begin{array}{c}£ \\
\ldots \\
\ldots \\
120,000 \\
173,000 \\
185,000 \\
194,000 \\
224,000 \\
251,000 \\
277,000\end{array}$ & $\begin{array}{c}£ \\
\mathbf{3 4}, 000 \\
138,000 \\
140,000 \\
141,000 \\
144,000 \\
147,000 \\
159,000 \\
170,000 \\
178,000\end{array}$ & $\begin{array}{c}£ \\
264,000 \\
964,000 \\
1,110,000 \\
1,190,000 \\
1,230,000 \\
1,269,000 \\
1,441,000 \\
1,566,000 \\
1,687,000\end{array}$ & $\begin{array}{c}£ \\
138,000 \\
18 \hat{6}, 000 \\
192,000 \\
196,000 \\
200,000 \\
204,000 \\
221,000 \\
236,000 \\
252,000\end{array}$ & $\begin{array}{c}£ \\
402,000 \\
1,150,000 \\
1,302,000 \\
1,386,000 \\
1,430,000 \\
1,473,000 \\
1,662,000 \\
1,802,000 \\
1,939,000\end{array}$ \\
\hline \multicolumn{9}{|c|}{ Men (Voluntary). } \\
\hline $\begin{array}{l}1912-13 \\
1913-14 \\
1914-15 \\
1915-16 \\
1916-17 \\
1917-18 \\
1922-23 \\
1927-28 \\
1932-33\end{array}$ & $\begin{array}{r}3,000 \\
13,000 \\
13,000 \\
13,000 \\
12,000 \\
12,000 \\
12,000 \\
12,000 \\
11,000\end{array}$ & $\begin{array}{l}2,000 \\
3,000 \\
3,000 \\
3,000 \\
3,000 \\
3,000 \\
2,000 \\
2,000 \\
2,000\end{array}$ & $\begin{array}{r}5,000 \\
21,000 \\
21,000 \\
22,000 \\
23,000 \\
23,000 \\
23,000 \\
23,000 \\
23,000\end{array}$ & $\begin{array}{r}\ldots \\
\ldots, 000 \\
8,000 \\
9,000 \\
9,000 \\
10,000 \\
12,000 \\
12,000\end{array}$ & $\begin{array}{l}\dddot{4}, 000 \\
6,000 \\
6,000 \\
6,000 \\
6,000 \\
5,000 \\
3,000 \\
3,000\end{array}$ & $\begin{array}{l}10,000 \\
41,000 \\
48,000 \\
52,000 \\
53,000 \\
53,000 \\
52,000 \\
52,000 \\
51,000\end{array}$ & $\begin{array}{l}6,000 \\
8,000 \\
8,000 \\
8,000 \\
8,000 \\
8,000 \\
7,000 \\
7,000 \\
6,000\end{array}$ & $\begin{array}{l}16,000 \\
49,000 \\
56,000 \\
60,000 \\
61,000 \\
61,000 \\
59,000 \\
59,000 \\
57,000\end{array}$ \\
\hline \multicolumn{9}{|c|}{ Women (Compulsory). } \\
\hline $\begin{array}{l}1912-13 \\
1913-14 \\
1914-15 \\
1915-16 \\
1916-17 \\
1917-18 \\
1922-23 \\
1927-28 \\
1932-33\end{array}$ & $\begin{array}{r}30,000 \\
124,000 \\
128,000 \\
129,000 \\
133,000 \\
136,000 \\
147,000 \\
159,000 \\
169,000\end{array}$ & $\begin{array}{l}19,000 \\
26,000 \\
27,000 \\
28,000 \\
28,000 \\
28,000 \\
31,000 \\
33,000 \\
35,000\end{array}$ & $\begin{array}{r}31,000 \\
132,000 \\
137,000 \\
141,000 \\
144,000 \\
147,000 \\
165,000 \\
180,000 \\
192,000\end{array}$ & $\begin{array}{c}\cdots \\
30,000 \\
4,4,000 \\
47,000 \\
50,000 \\
57,000 \\
67,000 \\
75,000\end{array}$ & $\begin{array}{l}13,000 \\
18,000 \\
18,000 \\
19,000 \\
19,000 \\
19,000 \\
20,000 \\
24,000 \\
24,000\end{array}$ & $\begin{array}{r}93,000 \\
300,000 \\
340,000 \\
361,000 \\
371,000 \\
380,000 \\
420,000 \\
463,000 \\
495,000\end{array}$ & $\begin{array}{r}\mathbf{5 6 , 0 0 0} \\
78,000 \\
79,000 \\
82,000 \\
83,000 \\
84,000 \\
89,000 \\
96,000 \\
102,000\end{array}$ & $\begin{array}{l}149,000 \\
378,000 \\
419,000 \\
443,000 \\
454,000 \\
464,000 \\
509,000 \\
599,000 \\
597,000\end{array}$ \\
\hline \multicolumn{9}{|c|}{ Women (Voluntary). } \\
\hline $\begin{array}{l}1912-13 \\
1913-14 \\
1914-15 \\
1915-16 \\
1916-17 \\
1917-18 \\
1922-23 \\
1927-28 \\
1932-33\end{array}$ & $\begin{array}{l}1,800 \\
7,400 \\
7,400 \\
7,400 \\
7,300 \\
7,300 \\
7,500 \\
7,800 \\
8,100\end{array}$ & $\begin{array}{l}1,200 \\
1,600 \\
1,600 \\
1,500 \\
1,500 \\
1,600 \\
1,500 \\
1,600 \\
1,700\end{array}$ & $\begin{array}{r}2,200 \\
9,000 \\
9,200 \\
9,300 \\
9,500 \\
9,700 \\
9,900 \\
10,500 \\
11,500\end{array}$ & $\begin{array}{c}\ldots \\
\ldots \\
3,300 \\
4,800 \\
\mathbf{5 , 2 0 0} \\
5,500 \\
6,100 \\
6,700 \\
8,500\end{array}$ & $\begin{array}{l}\cdots \\
\cdots \\
\cdots \\
\cdots \\
\cdots \\
\cdots \\
\cdots \\
\cdots \\
\cdots\end{array}$ & $\begin{array}{r}5,200 \\
18,000 \\
21,500 \\
23,000 \\
23,500 \\
24,100 \\
25,000 \\
26,600 \\
29,800\end{array}$ & $\begin{array}{l}3,400 \\
4,700 \\
4,600 \\
4,600 \\
4,500 \\
4,500 \\
4,400 \\
4,500 \\
4,600\end{array}$ & $\begin{array}{r}8,600 \\
22,700 \\
26,100 \\
27,600 \\
28,000 \\
28,600 \\
29,400 \\
31,100 \\
34,400\end{array}$ \\
\hline
\end{tabular}


TABLE XI.

Total Amount required in each of the under-mentioned financial years to meet the cost of the various "Minimum Benefits."

IRELAND.

Men (Compulsory).

\begin{tabular}{|c|c|c|c|c|c|c|c|}
\hline \multirow{2}{*}{ Year } & \multicolumn{4}{|c|}{ Benefits } & \multirow{2}{*}{$\begin{array}{c}\text { Total } \\
\text { Minimum } \\
\text { Beneht }\end{array}$} & \multirow{2}{*}{$\begin{array}{c}\text { Cost of } \\
\text { Adminis- } \\
\text { tration }\end{array}$} & \multirow{2}{*}{$\begin{array}{l}\text { Total } \\
\text { Outgo }\end{array}$} \\
\hline & $b$ & $c$ & $d$ & $e$ & & & \\
\hline $\begin{array}{l}1912-13 \\
1913-14 \\
1914-15 \\
1915-16 \\
1916-17 \\
1917-18 \\
1922-23 \\
1927-28 \\
1932-33\end{array}$ & $\begin{array}{c}£ \\
23,000 \\
31,000 \\
32,000 \\
33,000 \\
34,000 \\
35,000 \\
38,000 \\
41,000 \\
43,000\end{array}$ & $\begin{array}{c}£ \\
55,000 \\
229,000 \\
238,000 \\
247,000 \\
256,000 \\
266,000 \\
317,000 \\
342,000 \\
365,000\end{array}$ & $\begin{array}{c}\mathcal{E} \\
\ldots \\
. . \\
70,000 \\
102,000 \\
111,000 \\
117,000 \\
134,000 \\
154,000 \\
168,000\end{array}$ & $\begin{array}{c}£ \\
16,000 \\
65,000 \\
66,000 \\
66,000 \\
67,000 \\
69,000 \\
73,000 \\
78,000 \\
82,000\end{array}$ & $\begin{array}{c}£ \\
94,000 \\
325,000 \\
406,000 \\
448,000 \\
468,000 \\
487,000 \\
562,000 \\
615,000 \\
\mathbf{6 5 8 , 0 0 0}\end{array}$ & $\begin{array}{r}\mathscr{f} \\
68,000 \\
93,000 \\
95,000 \\
97,000 \\
99,000 \\
101,000 \\
108,000 \\
114,000 \\
119,000\end{array}$ & $\begin{array}{c}£ \\
162,000 \\
418,000 \\
501,000 \\
545,000 \\
567,000 \\
588,000 \\
670,000 \\
729,000 \\
777,000\end{array}$ \\
\hline \multicolumn{8}{|c|}{ Men (Voluntary). } \\
\hline $\begin{array}{l}1912-13 \\
1913-14 \\
1914-15 \\
1915-16 \\
1916-17 \\
1917-18 \\
1922-23 \\
1927-28 \\
1932-33\end{array}$ & $\begin{array}{r}9,000 \\
11,000 \\
11,000 \\
11,000 \\
11,000 \\
11,000 \\
11,000 \\
11,000 \\
10,000\end{array}$ & $\begin{array}{r}\mathbf{2 3 , 0 0 0} \\
93,000 \\
94,000 \\
96,000 \\
98,000 \\
98,000 \\
104,000 \\
106,000 \\
108,000\end{array}$ & $\begin{array}{l}25,000 \\
35,000 \\
37,000 \\
39,000 \\
49,000 \\
58,000 \\
66,000\end{array}$ & $\begin{array}{l}20,000 \\
26,000 \\
24,000 \\
23,000 \\
22,000 \\
18,000 \\
15,000 \\
14,000\end{array}$ & $\begin{array}{r}32,000 \\
124,000 \\
156,000 \\
166,000 \\
169,000 \\
170,000 \\
182,000 \\
190,000 \\
198,000\end{array}$ & $\begin{array}{l}28,000 \\
33,000 \\
33,000 \\
33,000 \\
33,000 \\
33,000 \\
32,000 \\
30,000 \\
28,000\end{array}$ & $\begin{array}{r}60,000 \\
157,000 \\
189,000 \\
199,000 \\
202,000 \\
203,000 \\
214,000 \\
220,000 \\
226,000\end{array}$ \\
\hline \multicolumn{8}{|c|}{ Women (Compulsory). } \\
\hline $\begin{array}{l}1912-13 \\
1913-14 \\
1914-15 \\
1915-16 \\
1916-17 \\
1917-18 \\
1922-23 \\
1927-28 \\
1932-33\end{array}$ & $\begin{array}{l}13,000 \\
18,000 \\
19,000 \\
19,000 \\
20,000 \\
20,000 \\
21,000 \\
23,000 \\
23,000\end{array}$ & $\begin{array}{r}23,000 \\
97,000 \\
100,000 \\
103,000 \\
107,000 \\
110,000 \\
126,000 \\
134,000 \\
144,000\end{array}$ & $\begin{array}{l}29,000 \\
43,000 \\
45,000 \\
48,000 \\
56,000 \\
64,000 \\
71,000\end{array}$ & $\begin{array}{r}4,000 \\
13,000 \\
13,000 \\
13,000 \\
13,000 \\
13,000 \\
13,000 \\
13,000 \\
14,000\end{array}$ & $\begin{array}{r}40,000 \\
128,000 \\
161,000 \\
178,000 \\
185,000 \\
191,000 \\
216,000 \\
234,000 \\
252,000\end{array}$ & $\begin{array}{l}39,000 \\
53,000 \\
54,000 \\
57,000 \\
57,000 \\
58,000 \\
63,000 \\
65,000 \\
68,000\end{array}$ & $\begin{array}{r}79,000 \\
181,000 \\
215,000 \\
235,000 \\
242,000 \\
249,000 \\
279,000 \\
299,000 \\
320,000\end{array}$ \\
\hline \multicolumn{8}{|c|}{ Women (Voluntary). } \\
\hline $\begin{array}{l}1912-13 \\
1913-14 \\
1914-15 \\
1915-16 \\
1916-17 \\
1917-18 \\
1922-23 \\
1927-28 \\
1932-33\end{array}$ & $\begin{array}{l}1,300 \\
1,800 \\
1,800 \\
1,800 \\
1,900 \\
1,900 \\
1,900 \\
1,900 \\
1,800\end{array}$ & $\begin{array}{r}2,900 \\
12,000 \\
12,400 \\
12,800 \\
13,200 \\
13,500 \\
14,200 \\
14,100 \\
13,900\end{array}$ & $\begin{array}{r}\ldots \\
\ldots \\
4,900 \\
7,000 \\
7,500 \\
8,000 \\
8,900 \\
9,800 \\
11,000\end{array}$ & $\begin{array}{l}\cdot \\
\cdots \\
. .\end{array}$ & $\begin{array}{r}4,200 \\
13,800 \\
19,100 \\
21,600 \\
22,600 \\
23,400 \\
25,000 \\
25,800 \\
26,700\end{array}$ & $\begin{array}{l}3,800 \\
5,100 \\
5,200 \\
5,200 \\
5,300 \\
5,400 \\
5,400 \\
5,200 \\
4,800\end{array}$ & $\begin{array}{r}8,000 \\
18,900 \\
24,300 \\
26,800 \\
27,900 \\
28,800 \\
30,400 \\
31,000 \\
31,500\end{array}$ \\
\hline
\end{tabular}


TABLE XII.

Annual Expenditure for "Maximum" Benefts and Cost of Administration.

ENGLAND.

\begin{tabular}{|c|c|c|c|c|c|}
\hline \multirow{2}{*}{ Year } & \multicolumn{2}{|c|}{$\mathrm{M}_{\mathrm{EN}}$} & \multicolumn{2}{|c|}{ WOMEN } & \multirow{2}{*}{ Total } \\
\hline & Compulıory & Voluntary & Compulsory & Voluntary & \\
\hline $1912-13$ & $\frac{f}{2.914 .000}$ & $\underset{156,200}{\$}$ & $\frac{\mathbb{E}}{1.081 .000}$ & $\stackrel{\mathscr{L}}{54,700}$ & $\begin{array}{c} \pm \\
4.205900\end{array}$ \\
\hline $1913-14$ & $8,336,000$ & 477,500 & $2,865,000$ & 142,700 & $11,821,200$ \\
\hline $1914-15$ & $9,470,000$ & 551,400 & $3,199,000$ & 161,800 & $13,282,200$ \\
\hline $1915-16$ & $10,097,000$ & 578,500 & $3,381,000$ & 170,300 & $14,226,600$ \\
\hline $1916-17$ & $10,422,000$ & 586,600 & $3,473,000$ & 172,300 & $14,653,900$ \\
\hline $1917-18$ & $10,781,000$ & 591,600 & $3,569,000$ & 174,300 & $15,115,900$ \\
\hline $1922-23$ & $12,200,000$ & 601,600 & $3,962,000$ & 181,500 & $16,945,100$ \\
\hline $1927-28$ & $13,262,000$ & 610,700 & $4,327,000$ & $18 \overrightarrow{\overline{5}}, 600$ & $18,385,300$ \\
\hline $1932-33$ & $14,329,000$ & 614,300 & $4,707,000$ & 190,800 & $19,841,100$ \\
\hline
\end{tabular}

WALES.

\begin{tabular}{|r|r|r|r|r|r|}
\hline $1912-13$ & 176,000 & 9,800 & 62,000 & 3,100 & 250,900 \\
$1913-14$ & 522,000 & 30,000 & 162,000 & 8,100 & 722,100 \\
$1914-15$ & 593000 & 34,700 & 182,000 & 9,200 & 818,900 \\
$1915-16$ & 633,000 & 36,300 & 194,000 & 9,600 & 872,900 \\
$1916-17$ & 652.000 & 36,700 & 198,000 & 9,900 & 896,600 \\
$1917-18$ & 675,000 & 37,000 & 203,000 & 10,000 & 925,000 \\
$1922-23$ & 764,000 & 37,600 & 226,000 & 10,300 & $1,037,900$ \\
$1927-28$ & 830,000 & 38,100 & 247,000 & 10,500 & $1,125,600$ \\
$1932-33$ & 900,000 & 38,500 & 269,000 & 10,900 & $1,218,400$ \\
\hline
\end{tabular}

SCOTLAND.

\begin{tabular}{|c|c|c|c|c|c|}
\hline \\
\hline $1912-13$ & 428.000 & 17,000 & 163,000 & 9,400 & 617,400 \\
\hline 191 & $1,223,000$ & 52,000 & 414,000 & 25,000 & $1,714,000$ \\
\hline $1914-15$ & $1,385,000$ & 60,000 & $\mathbf{4 5 9 , 0 0 0}$ & 28,700 & $1,932,700$ \\
\hline $1915-16$ & $\mathbf{1}, 474,000$ & $64,(1000$ & 486,000 & 30,400 & 2,054400 \\
\hline $1916-17$ & $1,521,000$ & 65,000 & 498,000 & 30,800 & $2,114,800$ \\
\hline $1917-18$ & $1,567,000$ & 65,000 & 509,000 & 31,500 & $2,172,500$ \\
\hline $1922-23$ & $1,769,0 \cap 0$ & 63,000 & 558,000 & 32,300 & $2,422,300$ \\
\hline $1927-28$ & $1,917,000$ & 63,000 & 613,000 & 34,200 & $2,627,200$ \\
\hline $1932-33$ & $2,063,000$ & 61,000 & 654,000 & 37,800 & $2,815,800$ \\
\hline \multicolumn{6}{|c|}{ IRELAND. } \\
\hline $1912-13$ & 172,000 & 64,000 & $87,000)$ & 8,800 & 331,800 \\
\hline & 445,000 & 167,000 & 198,000 & 20,800 & 830,800 \\
\hline $1914-15$ & 533,000 & 201,000 & 236,000 & 26,700 & 996,700 \\
\hline 16 & 578,000 & 212,000 & 258,000 & 29,500 & $1,077,500$ \\
\hline-17 & 603,000 & 215,000 & 265,000 & 30,700 & $1,113,700$ \\
\hline $1917-18$ & 625,000 & 216,000 & 273,000 & 31,700 & $1,145,700$ \\
\hline $1922-23$ & 711,000 & 228,000 & 306,000 & 33,400 & $1,278,400$ \\
\hline $1927-28$ & 776,000 & 234,000 & 308,000 & 34,100 & $1,372,100$ \\
\hline 1932-33 & 826,000 & 240000 & 351,000 & 34,600 & $1,451,600$ \\
\hline \multicolumn{6}{|c|}{ UNITED KINGDOM. } \\
\hline 1912-13 & $3,690,000$ & 247 & 000 & 76,000 & $5,406,000$ \\
\hline & $10,526,000$ & 726,500 & $3,639,000$ & 196,600 & $15,088,100$ \\
\hline $1914-15$ & $11,981,000$ & 847,100 & $4,076,000$ & 226,400 & $17,130,500$ \\
\hline $1915-16$ & $12,782,000$ & 890,800 & $4,319,000$ & 239,800 & $18,231,600$ \\
\hline $1916-17$ & $13,198,000$ & 903,300 & $4,434,000$ & 243,700 & $18,779,000$ \\
\hline $1917-18$ & $13,648,000$ & 909,600 & $4,554,000$ & 247,500 & $19,359,100$ \\
\hline $1922-23$ & $15,444,4,000$ & 930,200 & $5,052,000$ & 257,500 & $21,683,700$ \\
\hline $1927-28$ & $16,785,000$ & 945,800 & $5,515,000$ & 264,400 & $23,510,200$ \\
\hline $1932-32$ & $18,118,000$ & 953,800 & $5,981,000$ & 274,100 & $25,326,900$ \\
\hline
\end{tabular}


TABLE XIII.

Annual Charge in respect of the State Proportion of "Maximum" Benefits and Cost of Administration.

ENGLAND.

\begin{tabular}{|c|c|c|c|c|c|c|}
\hline \multirow{2}{*}{ Year } & \multicolumn{2}{|c|}{ MEN } & \multicolumn{2}{|c|}{ WOMEN } & \multirow{2}{*}{ Sanatoria } & \multirow{2}{*}{ Total } \\
\hline & Compulsory & Voluntary & Compulsory & Voluntary & & \\
\hline $\begin{array}{l}1912-13 \\
1913-14 \\
1914-15 \\
1915-16 \\
1916-17 \\
1917-18 \\
1922-23 \\
1927-28 \\
1932-33\end{array}$ & $\begin{array}{c}£ \\
648,000 \\
1,852,000 \\
2,104,000 \\
2,243,000 \\
2,316,000 \\
2,396,000 \\
2,711,000 \\
2,947,000 \\
3,185,000\end{array}$ & $\begin{array}{c}£ \\
34,700 \\
106,100 \\
122,500 \\
128,600 \\
130,300 \\
131,500 \\
133,700 \\
135,700 \\
136,500\end{array}$ & $\begin{array}{r}\mathscr{L} \\
270,000 \\
716,000 \\
800,000 \\
845,000 \\
868,000 \\
892,000 \\
991,000 \\
1,082,000 \\
1,177,000\end{array}$ & \begin{tabular}{c|}
$f$ \\
13,700 \\
35,700 \\
40,500 \\
42,600 \\
43,100 \\
43,600 \\
45,400 \\
46,400 \\
47,700
\end{tabular} & \begin{tabular}{c|}
$£$ \\
31,600 \\
43,000 \\
43,800 \\
44,800 \\
45,700 \\
46,600 \\
51,000 \\
55,100 \\
59,100
\end{tabular} & $\begin{array}{c}£ \\
998,000 \\
2,752,800 \\
3,110,800 \\
3,304,000 \\
3,403,100 \\
3,509,700 \\
3932,100 \\
4,266,200 \\
4,605,300\end{array}$ \\
\hline \multicolumn{7}{|c|}{ WALES. } \\
\hline $\begin{array}{l}1912-13 \\
1913-14 \\
1914-15 \\
1915-16 \\
1916-17 \\
1917-18 \\
1922-23 \\
1927-28 \\
1932-33\end{array}$ & $\begin{array}{r}39,000 \\
116,000 \\
132,000 \\
141,000 \\
145,000 \\
150,000 \\
170,000 \\
185,000 \\
200,000\end{array}$ & $\begin{array}{l}2,200 \\
6,700 \\
7,700 \\
8,100 \\
8,200 \\
8,200 \\
8,400 \\
8,500 \\
8,600\end{array}$ & $\begin{array}{l}16,000 \\
41,000 \\
46,000 \\
49,000 \\
50,000 \\
51,000 \\
57,000 \\
62,000 \\
67,000\end{array}$ & $\begin{array}{r}800 \\
2,000 \\
2,300 \\
2,400 \\
2,500 \\
2,500 \\
2,600 \\
2,600 \\
2,700\end{array}$ & $\begin{array}{l}1,900 \\
2,600 \\
2,700 \\
2,700 \\
2,800 \\
2,800 \\
3,100 \\
3,400 \\
3,600\end{array}$ & $\begin{array}{r}59,900 \\
168,300 \\
190,700 \\
203,200 \\
208,500 \\
214,500 \\
241,100 \\
261,500 \\
281,900\end{array}$ \\
\hline \multicolumn{7}{|c|}{ SCOTLAND. } \\
\hline $\begin{array}{l}1912-13 \\
1913-14 \\
1914-15 \\
1915-16 \\
1916-17 \\
1917-18 \\
1922-23 \\
1927-28 \\
1932-33\end{array}$ & $\begin{array}{r}95,000 \\
272,000 \\
308000 \\
327,000 \\
338,000 \\
348,000 \\
393,000 \\
426,000 \\
458,000\end{array}$ & $\begin{array}{r}4,000 \\
12,000 \\
13,000 \\
14,000 \\
14,000 \\
14,000 \\
14,000 \\
14,000 \\
13,000\end{array}$ & $\begin{array}{r}41,000 \\
104,000 \\
115,000 \\
122,000 \\
125,000 \\
127,000 \\
140,000 \\
153,000 \\
164,000\end{array}$ & $\begin{array}{l}2,400 \\
6,300 \\
7,200 \\
7,600 \\
7,700 \\
7,900 \\
8,100 \\
8,600 \\
9,500\end{array}$ & $\begin{array}{l}4,700 \\
6,300 \\
6,400 \\
6,500 \\
6,700 \\
6,800 \\
7,400 \\
8,000 \\
8,500\end{array}$ & $\begin{array}{l}147,100 \\
400,600 \\
449,600 \\
477,100 \\
491,400 \\
503,700 \\
562,500 \\
609,600 \\
653,000\end{array}$ \\
\hline \multicolumn{7}{|c|}{ IRELAND. } \\
\hline $\begin{array}{l}1912-13 \\
1913-14 \\
1914-15 \\
1915-16 \\
1916-17 \\
1917-18 \\
1922-23 \\
1927-28 \\
1932-33\end{array}$ & $\begin{array}{r}51,000 \\
135,000 \\
155,000 \\
166,000 \\
172,000 \\
178,000 \\
201,000 \\
218,000 \\
233,000\end{array}$ & $\begin{array}{l}18,000 \\
50,000 \\
58,000 \\
60,000 \\
60,000 \\
61,0110 \\
63,000 \\
64,000 \\
65,000\end{array}$ & $\begin{array}{r}27,000 \\
73,000 \\
83,000 \\
90,000 \\
92,000 \\
94,000 \\
104,000 \\
112,000 \\
119,000\end{array}$ & $\begin{array}{r}2,800 \\
7,600 \\
9,000 \\
9,800 \\
10,100 \\
10,400 \\
10.900 \\
11,000 \\
11,000\end{array}$ & $\begin{array}{l}3,100 \\
4,200 \\
4,300 \\
4,400 \\
4,400 \\
4,500 \\
4,800 \\
5,100 \\
5,300\end{array}$ & $\begin{array}{l}101,900 \\
269,800 \\
309300 \\
330,200 \\
338,500 \\
347,900 \\
383,700 \\
410,100 \\
433,300\end{array}$ \\
\hline
\end{tabular}




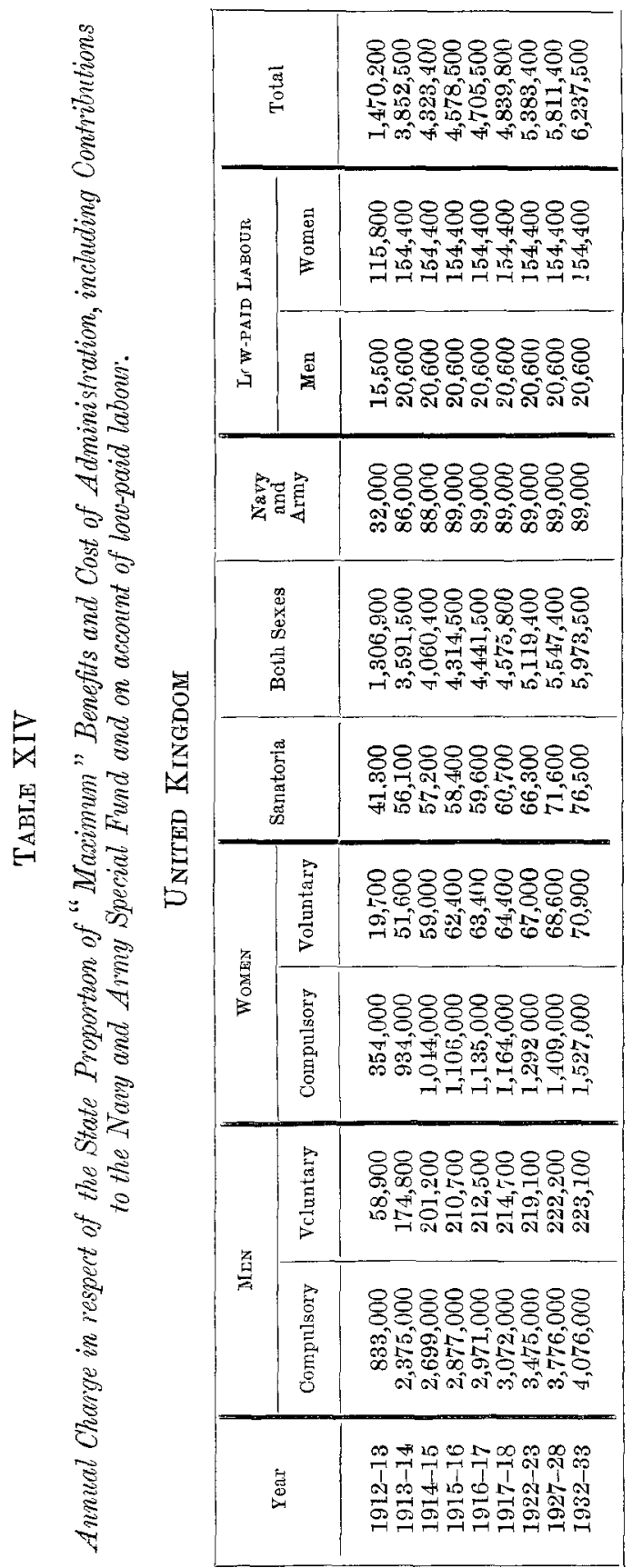

